\title{
Lymph Node Metastases of Prostatic Adenocarcinoma in the Mesorectum in Patients with Rectal Cancer
}

\author{
In Ja Park, M.D., Hee Cheol Kim, M.D., Chang Sik Yu, M.D., Choung Soo Kim, M.D. ${ }^{1}$, Jung Sun Kim, \\ M.D. ${ }^{2}$ and Jin Cheon Kim, M.D. \\ Departments of Surgery, ${ }^{2}$ Urology and ${ }^{1}$ Pathology, University of Ulsan College of Medicine and Colorectal Clinic, Asan \\ Medical Center, Seoul, Korea
}

Lymph node involvement is the most important prognostic factor of rectal cancer. Cancer originating from sites other than the rectum rarely metastasizes to the mesorectal lymph node. We report a rectal cancer patient

\section{INTRODUCTION}

Lymph node metastases are an important prognostic factor following surgery for rectal cancer. Therefore, meticulous examination of the mesorectal lymph node is essential. Metastasis to the mesorectal lymph node from other sites, with the exception of the anus and rectum, is very rare. Prostatic cancer is the second most common cancer in males in Western countries. Despite the incidence of prostatic adenocarcinomas, the propensity for metastatic spread and reports on the unusual locations of lymph node metastases are very rare (1). In addition, mesorectal lymph node metastasis from sites other than the rectum has rarely been reported (2). We report a case of rectal cancer in a patient with synchronous metastatic prostatic cancer in the mesorectal lymph node. This case report appears to be the second report of lymph node metastasis to the mesorectum from a prostatic adenocarcinoma documented in the English literature.

\section{CASE REPORT}

A 76-year-old man presented with a 2-month history of constipation and defecation difficulty. He also complained of voiding difficulty. He had no family history of colon or related cancer. On manual rectal examination, a hard mass, without a mucosal lesion, was palpated on the ventral side. CT scans showed an encircling mass, with perirectal infiltration at the rectosigmoid junction and a mass at the prostate (Fig. 1). The patient subsequently underwent a colonoscopy, which revealed

Correspondence: Jin Cheon Kim, Department of Surgery, University of Ulsan College of Medicine, 388-1, Pungnap-dong, Songpa-gu, Seoul 138-736, Korea. (Tel) 82-2-3010-3489, (Fax) 82-2-474-9027,

(E-mail) jckim@amc.seoul.kr

Received March 16, 2005, Accepted March 25, 2005 with a synchronous metastatic prostatic carcinoma to the mesorectal lymph node. (Cancer Res Treat. 2005;37:129-132)

Key Words: Mesorectal, Lymph node, Prostatic carcinoma

an obstructing mass in the rectosigmoid area (Fig. 2). The biopsy performed on the rectal mass showed an adenocarcinoma, with moderate differentiation. Because of the mass lesion at the prostate on CT scan, a needle biopsy was performed for this lesion. The needle biopsy revealed a prostatic adenocarcinoma (Fig. 3A). The patient underwent a low anterior resection for rectal cancer, and was consulted to a urologic surgeon for the prostatic lesion, who decided to perform postoperative radiotherapy and hormonal therapy.

A $5.3 \mathrm{~cm}$ sized encircling ulcerofungating mass was present in the low anterior resection specimen. This was a typical colonic adenocarcinoma, moderately differentiated, invading the entire rectal wall, with accompanying metastasis to the lymph nodes. The lymph nodes showed metastasis of another adenocarcinoma, with a different microscopic pattern. The tumor was composed of cuboidal or columnar cells, with clear pale cytoplasm and round nuclei in a small glandular, cribriform or diffuse infiltrative pattern (Gleason grade 9). From immunostaining, they were positive for PSA and PAP, prostatic markers, while the colonic adenocarcinoma cells were negative for these markers (Fig. 3).

$\mathrm{He}$ recovered uneventfully. After recovery, he underwent radiotherapy and hormonal therapy for prostatic cancer, and has been followed up without evidence of recurrence for 15 months.

\section{DISCUSSION}

The prostate is richly supplied with lymphatics, which drain into the obturator-hypogastric and presacral nodes (3). Micrometastasis to these lymph nodes is known as a probably early and frequent event, with a clinically localized carcinoma of the prostate detected with high incidence involving a node in these two groups (4). In contrast, as far as we could determine in a search of the literature, lymph node metastasis of a prostatic carcinoma to the mesorectum has been reported only once before (2).

In our case, PSA and PSAP were both strongly positive in the tumor cells of the lymph node. Staining of nonprostatic 

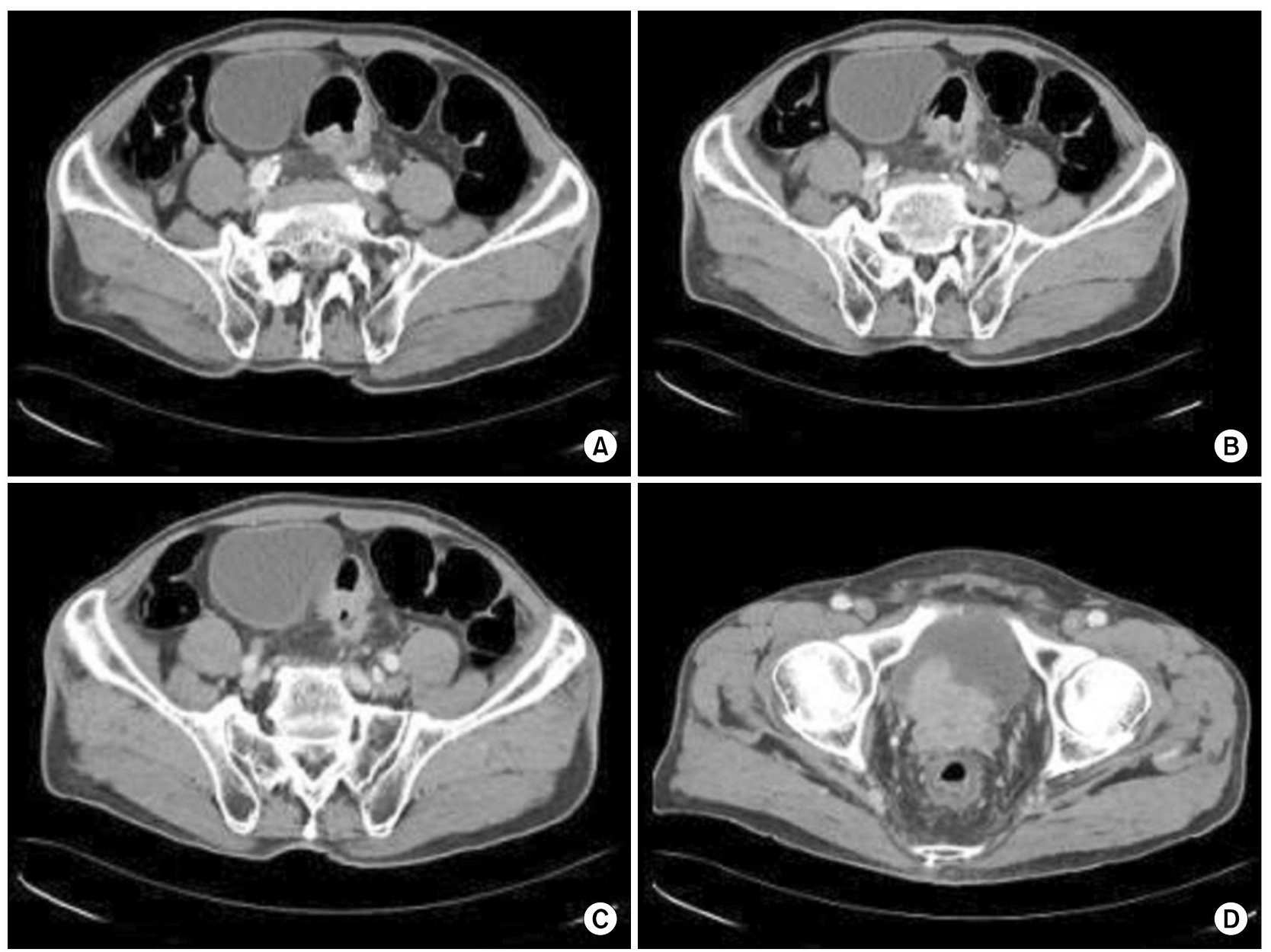

Fig. 1. CT scan shows the encircling mass at the rectosigmoid junction. (A, B, C) An obstructing mass in the distal sigmoid colon. (D) Mass lesion in the prostate.

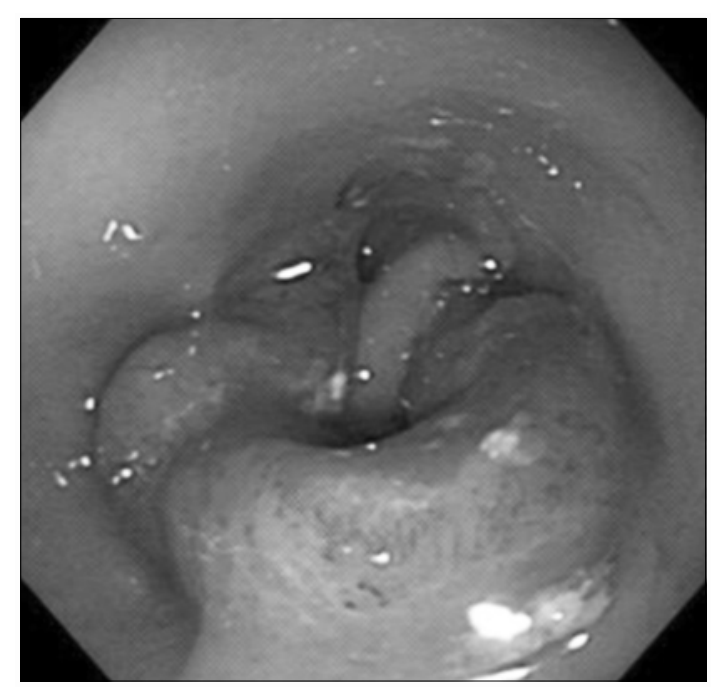

epithelial cells for PSA has been reported $(5,6)$; however, it was found that these false staining were caused by a batch of faulty antiserum (7).

These observations of lymph node metastasis of a prostatic adenocarcinoma in the mesorectum show that a lymphatic connection could exist between hypogastric lymphatic drainage and mesorectal drainage. Actually, an extension of a rectal adenocarcinoma to extra-mesenteric lymph nodes was documented many years previously (8). Therefore, lateral node dissection has been advocated, even for advanced rectal carcinoma, at or below the peritoneal reflection (9). Moreover, there is no reason to believe that such drainage would be preferentially from the prostate to the mesorectum, rather than from the rectum to extramesenteric lymph nodes. Such a hypothesis would partially explain the high numbers of local recurrence found in cases of low rectal cancer (10).

Fig. 2. Colonoscopy reveals the luminal-obstructing lesion in the distal sigmoid area. 

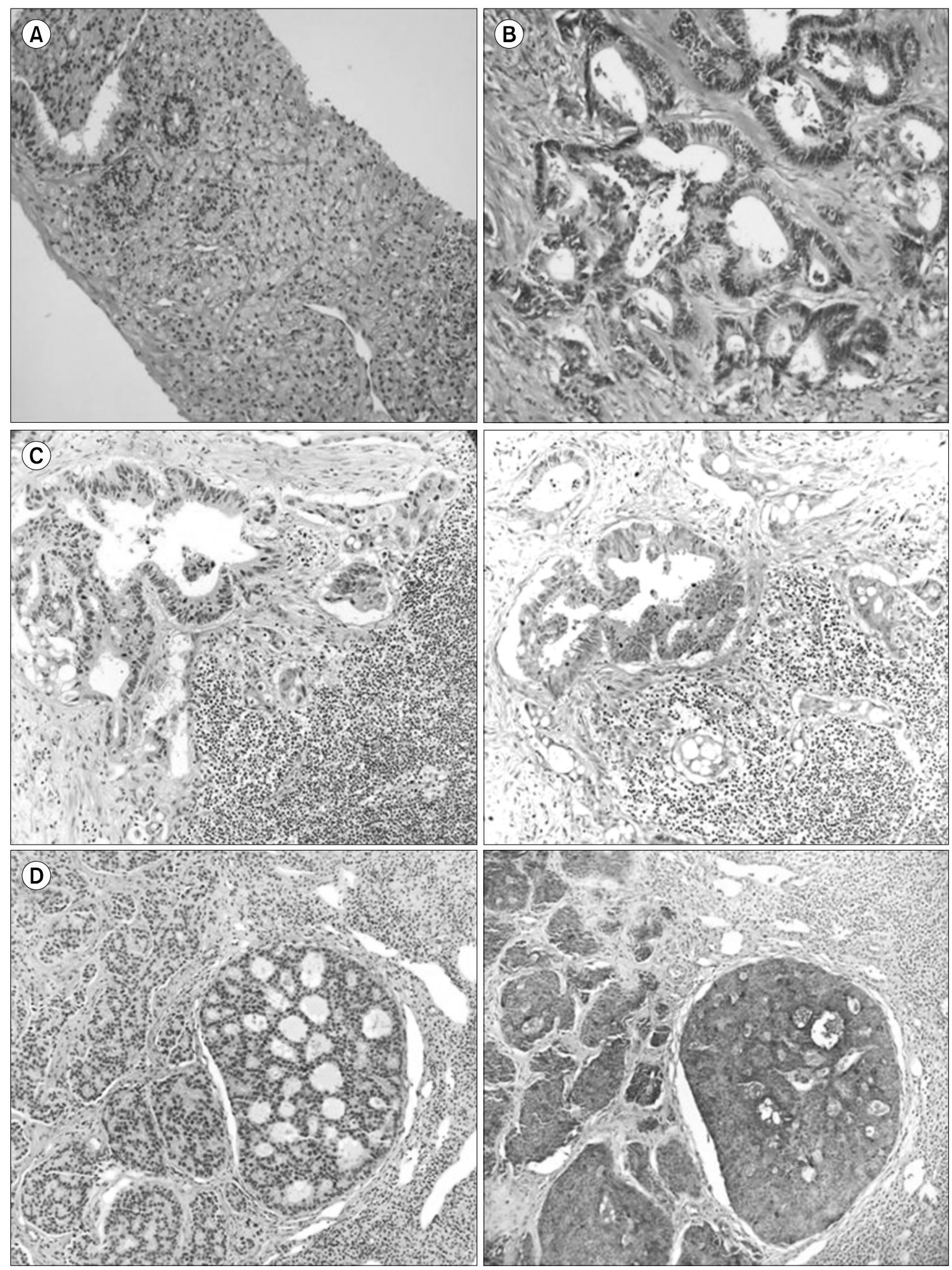

Fig. 3. (A) The adenocarcinoma in the prostate, composed of cuboidal or columnar clear cells with a cribriform pattern. (B) The adenocarcinoma in the colon, with a complex glandular structures and pleomorphic columnar cells. (C) Right; The colonic adenocarcinoma metastasing to a lymph node. Left; The colonic adenocarcinoma is negative for PAP on immunostaining. (D) Right; The prostatic adenocarcinoma metastasizing to a lymph node. Left; The prostatic adenocarcinoma is positive for PAP on immunostaining. 


\section{REFERENCES}

1. Jones H, Anthony PP. Metastatic prostatic carcinoma presenting as left-sided cervical lymphadenopathy: a series of 11 cases. Histopathology. 1992;21:149-54.

2. Mourra N, Pare Y, McNamara D, Tiret E, Flejou JF, Parc R. Lymph node metastases of prostatic adenocarcinoma in the mesorectum in patients with adenocarcinoma or villous tumor of the rectum with collision phenomenon in a single lymph node: report of five cases. Dis Colon Rectum. 2005; online first

3. Smith MJ. The lymphtics of the prostate. Invest Urol. 1966;3: 439-44.

4. McLaughlin AP, Saltzstein SL, McCullough DL, Gittes RF. Prostatic carcinoma: incidence and location of unsuspected lymphatic metastasis. J Urol. 1976;115:89-94.
5. Wibur DC, Krenaer K, Bonfiglio TA. Prostatic specific antigen (PSA) staining in carcinoma of non prostatic origin. Am J Clin Pathol. 1987;12:530.

6. May EE, Perentes E. Anti-Leu 7 immunoreactivity with human tumors: its value in the diagnosis of prostatic adenocarcinoma. Histopathology. 1987;11:295-304.

7. Herman E, Elfont E, Boenisch T. Prostate-specific antigen. Histopathology. 1988;12:687-8.

8. Stearns MW, Jr. Deddish MR. Five-year results of abdominopelvic lymph node dissection for carcinoma of the rectum Dis Colon Rectum. 1959;2:169-72.

9. Moriya Y, Hojo K, Sawada T, Koyama Y. Significance of lateral node dissection for advanced rectal carcinoma at or below the peritoneal reflection. Dis Colon Rectum. 1989;32: 307-15.

10. Kapiteijn E, Marijnen CA, Nagtegaal ID, Putter H, Steup WH, Wiggers T, et al. Preoperative radiotherapy combined with total mesorectal excision for respectable rectal cancer. N Engl J Med. 2001;345:638-46. 